\title{
A comparative study of chart 1 and chart 7 of amsler grid in early detection of symptomatic diabetic macular edema
}

\author{
Nilesh Shrimali ${ }^{1}$, Minhaz Karkhanawala ${ }^{2}$, Somesh Aggarwal $^{3 *}$, Uma Gajiwala ${ }^{4}$, Pratik katariya ${ }^{5}$ \\ 1,4,5 Resident, ${ }^{2}$ Senior Resident, ${ }^{\mathbf{3}}$ Professor and Head of Retina Unit, M and J Western Regional Intitute of Ophthalmology Ahmedabad, \\ Gujarat, India
}

\begin{abstract}
Objective: The aim of this study is to compare the sensitivities of chart 1 and chart 7 of the Amsler grid in early detection of metamorphopsia suggestive of diabetic macular edema.

Design: It was a randomized, prospective, analytical study. Eighty patients were divided into two equal groups- A and B. Group A patients were given Amsler chart 1 and group B patients given Amsler chart 7.Regular 3 monthly follow up was done for 2 years with Optical Coherence Tomography (OCT) to detect DME on every follow up.

Setting: Retina Unit, M And J Western Regional Institute Of Ophthalmology, Civil Hospital, Asarwa, Ahmedabad, Gujarat, India.

Subjects: 80 eyes of 80 patients were enrolled, equally divided into 2 groups of 40 patients each.

Intervention: No intervention was done for purpose of study. DME was treated as per clinical guidelines.

Main outcome and Measures: Sensitivity and Specificity of both Amsler chart were calculated and compared using statistical analysis.

Results: The sensitivity of Chart 1 of Amsler grid in detecting metamorphopsia was 56.25 percent, whereas the sensitivity of Chart 7 of Amsler grid was 76.47 percent. The difference was statistically significant $(\mathrm{P}<0.05)$. The specificity of Amsler test in both groups was 100 percent.
\end{abstract}

Conclusion: Amsler grid type 7 is a superior tool in detecting metamorphopsia due to macular edema than type 1 conventional Amsler grid.

Keywords: Amsler grid, Diabetic macular edema.

\section{Introduction}

India is becoming the world capital of diabetesmellitus and diabetic retinopathy as its sequel is one of the most common conditions contributing to visual morbidity. Rapid progression of senile cataract, diabetic macular edema and complications of proliferative diabetic retinopathy are some of the common causes responsible for vision loss in patients with long standing diabetes. Early detection of these can help the physician and the ophthalmologist in preventing their rapid progression and beginning early treatment which limits long term visual disability. Diabetic macular edema is a complication that is severely disabling for the patient due to affection of central vision. For detection of visual abnormalities due to diabetic macular edemaat an early stage, several methods have evolved, majority of which require the visit to an ophthalmologist. These methods include regular testing of visual acuity and detecting metamorphopsia by subjective testing using the Amsler grid and M-charts invented by Matsumoto. This is because metamorphopsia is the most important and earliest symptom of macular edema and can be detected earlier than decrease in visual acuity. Amsler grid is the gold standard method to detect metamorphopsia at an early stage and has been used since 1947. The advantage of this method is that it is easy to perform, cheap, safe and can be done by the patient at home; thus making the patient an active participant in the management of his illness. Earlier detection of diabetic macular edema in this manner can enable a quicker onset of treatment for the affected eye. The aim of this study is to compare the sensitivities of chart 1 and chart 7 of the Amsler grid in early detection of metamorphopsia suggestive of diabetic macular edema.

\section{Materials and Methods}

Eighty $(80)$ eyes of 80 patients from the diabetic clinic of the retina department of the tertiary hospital were enrolled in the studybetween January 2015 to June2016. It was arandomized, prospective, analytical study. Informed written consent was taken from each participant after explaining the nature of the study. The inclusion criteria were subjects having diabetes mellitus for more than 5 years, patients having only mild NPDR without macular edema, visual acuity of N/6 on Roman test types and patients who voluntarily consented for inclusion in the study. Patients who were already having macular edema or advanced disease, patients having hypertension and other possible causes of macular edema, patients who had undergone cataract or other eye surgeries and patients more than 60 years of age were excluded.

Patients were randomly divided in to two groups-Group A and Group B. Patients in Group A were given Chart 1 of Amsler grid and those in Group B were given chart 7 of Amsler grid. All the patients were asked to look for metamorphopsia daily and those who detected

\footnotetext{
*Corresponding Author: Somesh Aggarwal, M and J Western Regional Intitute of Ophthalmology Ahmedabad, Gujarat, India
} Email:dr.somesh@yahoo.com http://doi.org/ 10.18231/j.ijooo.2019.035 
metamorphopsia were asked to report immediately. The test was considered positive if any deviation in lines was reported by the patient. All patients were also asked to come for a regular check up every 3 months for a period of two years. On each follow up examination, patients were examined with Slit Lamp Biomicroscopy with 90D lens, Fundus Flourescein Angiography(FFA) and Spectral Domain- Optical Coherence Tomography (SD-OCT).These findings were compared with the reported Amsler chart findings.

At the end of two years follow up, the data was analyzed, sensitivity of Amsler test in group A and group B was compared and statistical analysis done using Fisher's exact test. Detection of macular edema on SD-OCT was taken as gold standard and sensitivity of detection of metamorphopsia was compared against it.

$P$ value less than 0.05 indicated statistical significance.

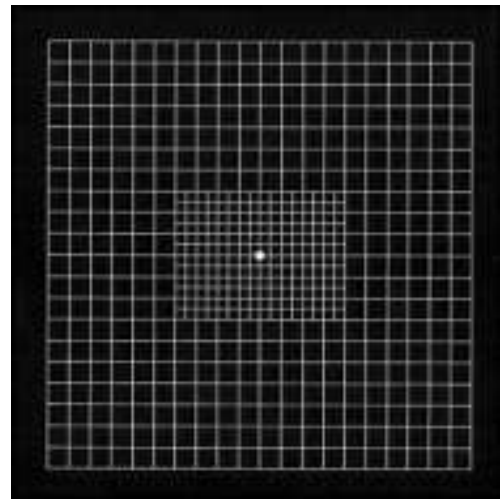

Fig. 1: Amsler chart 1

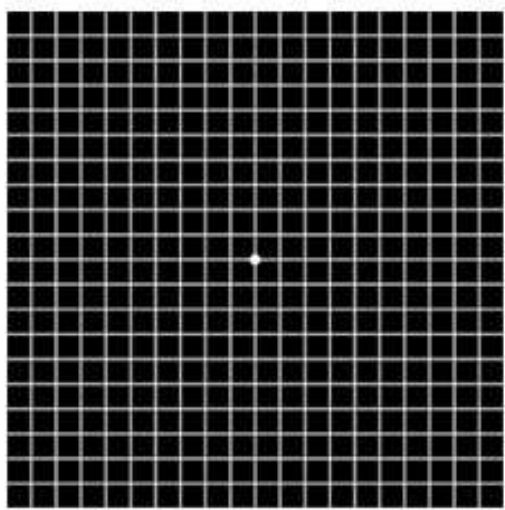

Fig. 2: Amsler chart 7

\section{Results}

Group A consisted of 24 male and 16 female patients with age ranging from 42.1 years to 58.3 years (mean age 51.4 years)

Group B consisted of 22 male and 18 female patients with age ranging from 43.2 years to 59.5 years (mean age 50.6 years)

It was observed that out of 40 patients of group A, 18 reported metamorphopsia in a follow up of $2 \mathrm{yrs}(45 \%)$; while in group B, out of 40 patients, 26 reported metamorphopsia (65\%). On SD-OCT, at the end of two years, 32 patients in group A were detected to have macular edema $(80 \%)$ during the study period while in group B, 34 patients were detected with macular edema $(85 \%)$.

In group $\mathrm{A}$, all the patients who reported metamorphopsia were having macular edema on OCT but in addition to that 14 more patients had macular edema on OCT. Similarly in group B, though 26 patients had metamorphopsia with all patients having macular edema on OCT, 8 more patients had macular edema that was not reported by the patient.

The sensitivity of Chart 1 of Amsler grid in detecting metamorphopsia was 56.25 percent, whereas the sensitivity of Chart 7 of Amsler grid was 76.47 percent. The difference was statistically significant $(\mathrm{P}<0.05)$. The specificity of Amsler test in both groups was 100 percent.

Table 1: Detection of diabetic macular edema by amsler test and Oct

\begin{tabular}{|l|c|c|}
\hline \multicolumn{1}{|c|}{ Groups } & \multicolumn{1}{c|}{$\begin{array}{c}\text { Amsler Test } \\
\text { Positive }\end{array}$} & $\begin{array}{c}\text { DME on } \\
\text { Oct. }\end{array}$ \\
\hline $\begin{array}{l}\text { Group A } \\
\text { (40 patients) }\end{array}$ & $18(45 \%)$ & $32(80 \%)$ \\
\hline $\begin{array}{l}\text { Group B } \\
\text { (40 patients) }\end{array}$ & $26(65 \%)$ & $34(85 \%)$ \\
\hline
\end{tabular}

\section{Discussion}

OCT has evolved to become a very sensitive investigation and the gold standard in detecting diabetic macular edema. It further decides the type of macular edema and accordingly guides management. However, all hospitals, especially those in the periphery are not equipped with the machine and equipments required for OCT. In developing countries, this problem gets compounded by the fact that patients present to tertiary hospitals with advanced disease, and consequentially suffer from long term complications of chronic macular edema. The Amsler grid can play a significant role in reducing this time to presentation and can involve the patient in management of his or her own illness. It is an inexpensive, simple and reliable method to detect metamorphopsia and hence diagnose diabetic macular edema early. By suggesting need for OCT in symptomatic patients, it also promotes judicious and efficient use of OCT, as cost is always a concern in developing countries like India.

The chart 1 of the Amsler grid is made up of a white grid against a black background.The grid encloses 400 smaller $0.5 \mathrm{~mm}$ squares which when viewed from about a distance of one third of a meter; eachsquare subtends anangle of 1 degree. Chart 7 of Amsler grid includes a still finer central grid, with each square subtending an angle of half a degree.

In a study by Moustafa et al. the sensitivity of detection of macular diseases by Amsler grid was 88\% (95\% confidence interval: 77.9-97.6\%). There was substantial agreement between results of Amsler test and those of FFA and OCT. ${ }^{1}$ 
In this study, the sensitivity of Amsler chart 1 was found to be 56.25 percent and that of Amsler chart 7 was found to be 76.47 percent. This clearly shows that Amsler chart 7 is much more sensitive than chart 1 in earlier detection of metamorphopsia; however the sensitivity of the test is less compared to that in the study by Moustafa et al.

Several other attempts have been made to improve the sensitivity of Amsler testing. In a study by Augustin et al., the investigators compared the original chart 1 of the Amslerwith a modified Amsler grid presenting black lines on a white background. 182 eyes were studied to detect metamorphopsia in patients with age related macular degeneration. However, the original Amsler grid provided significantly better results than the modified version. ${ }^{2}$

Threshold Amsler testing involves asking the patient to view the white on black version of the chart through polarizing filters, reducing the luminance of the grid. This has been shown to improve scotoma detection in patients with diabetic retinopathy. ${ }^{3}$

In another study where the standard and threshold Amsler grid testing was compared with standard and threshold fundus perimetry, $77 \%$ of standard and $87 \%$ of threshold scotomas were not detected by Amsler grid testing. Also, more than half of the distortion reported was at the retinal areas corresponding to the scotoma area, not a nonscotoma retinal area. ${ }^{4}$

Another modification of the Amsler chart which has been reported is to present it as a blue grid on a yellow background, or in various other colors. ${ }^{5}$ No data on the sensitivity for these charts have been published in the peer reviewed literature.

When compared with the preferential hyperacuity perimeter (PHP), the perimeter was found to be more sensitive in detection of choroidal neovascularization in patients with ARMD than Amsler test. The PHP is a computer based screening test, hence can be used only if the patient owns a PC. ${ }^{6}$

A new modality- the Macular Computerized Psychophysical test (MCPT) has been found superior to the Amsler grid in detecting AMD-related lesions and is being investigated to determine its feasibility for home monitoring to provide early detection to $\mathrm{CNV}$. $^{7}$

A further computer based screening test which has been suggested is the Macular mapping test, yet sensitivity and specificity data on this test are yet to be published in peer reviewed literature. ${ }^{8}$

A novel tool for measuring metamorphopsia was developed using commercial 3D display at Seoul, South Korea, which demonstrated a sensitivity of $100 \%$ and specificity of $90.7 \%$ as compared to sensitivity of $66.7 \%$ on Amsler grid test. The test was based on three-dimensional stereoscopic monitor during straight line testing of both eyes. ${ }^{9}$
The search for a simple, cheap, easy to use screening test which the patientscan use in their own home, which is also highly sensitive and specific for detection of macular edema, still goes on. Such a system would help early identification and presentation of patients with macular disease, with potentially large public health benefits.

\section{Conclusion}

Amsler grid type 7 is a superior tool in detecting metamorphopsia due to macular edema than type 1 conventional Amsler grid.More studies are required to establish the usefulness of this in the early detection of macular edema as this is simple and inexpensive method; along with constant search for a better tool with higher sensitivity.

\section{Source of Funding}

None.

\section{Conflicts of Interest}

None

\section{Reference}

1. Moustafa KN, Nermeen MB, Mostafa MMD. Resurrection of the Amsler chart in macular diseases. 2015;28(1): 174-80.

2. Augustin AJ, Offermann JL, Lutz J, Schmidt-Erfurth U, Tornambe P. Comparison of the original Amsler grid with the modified Amsler grid: Result for patients with Age Related Macular Degeneration. Retina 2005;25(4): 443-5.

3. Wolfe KA, Sadun AA. Threshold Amsler grid testing in diabetic retinopathy. Graefes Arch Clin Ophthalmol 1991;229(3):219-23

4. Schuchard RA. Validity and Interpretation of Amsler grid reports. Arch Ophthalmol 1993;111(6):776-80.

5. Mutlukan E. Red dots visual field test with blue on yellow and blue on red macula test grid. Eye(Lond) 2006;20(4):506-8

6. David L.C.I., Marcos P.A., Arnaldo P.C. Comparison of the original Amsler grid with the preferential hyperacuity perimeter for detecting choroidal neovascularization in agerelated macular degeneration. Arq Bras Ophthalmol 2007;70(5).

7. Loewenstein A, Malach R, Goldstein M, Leibovitch I, Barak A, Baruk E, et al. Replacing the Amsler grid. A new method for monitoring patients with age related maculardegeneration. Ophthalmol 2003;110(5):966-70.

8. Trauzetterl-Klosinski S, Biermann P, Hahn G. Assessment of parafoveal function in maculopathy: a comparison between the Macular Mapping Test and kinetic Manual Perimetry. Graefes Arch Clin Exp Ophthalmol 2003;241(12): 988-95

9. Ji Won Kim, Yun TK. Clinical application of 3D display device in ophthalmology: Measurement of metamorphopsia. Acta Ophthalmol 2015;94(6).

How to cite this article: Shrimali N, Karkhanawala M, Aggarwal S, Gajiwala U, katariya P, A comparative study of chart 1 and chart 7 of amsler grid in early detection of symptomatic diabetic macular edema, Int J Ocul Oncol Oculoplasty 2019;5(3):136-8. 